\title{
TÁC ĐộNG CỦA ĐẠI SƯ THƯƠNG HIỆU LÊN ĐẾN Ý ĐỊNH MUA CỦA KHÁCH HÀNG TẠI THÀNH PHỐ HỒ CHÍ MINH
}

\author{
NGUYẼ̃N QUỐC CƯỜNG, VÕ THI XUÂN QUỲNH, NGUYẼ̃N ANH PHÚC \\ Khoa Quản Trị Kinh Doanh, Truờng Đại học Công nghiệp thành phố Hồ Chí Minh; \\ nguyenquoccuong@iuh.edu.vn,
}

Tóm tắt: Mục đích của nghiên cứu này nhằm phân tích những yếu tố của đại sứ thương hiệu tác động đến ý định mua của khách hàng tại khu vực Thành phố Hồ Chí Minh, với số lượng mẫu nghiên cứu là 210 người đang sinh sống tại Thành phố Hồ Chí Minh, độ tuổi từ 16 và lớn hơn, khảo sát được thực hiện thông qua bảng câu hỏi trực tuyến. Nhóm tác giả tiến hành phân tích hồi quy tuyến tính với ý định mua hàng là biến phụ thuộc và 6 biến độc lập có nguồn gốc từ các tài liệu tham khảo, gồm: Sự thu hút, sự tin cậy, chuyên môn, sự quen thuộc, sự phù hợp của người nổi tiếng với thương hiệu/sản phẩm và các thông tin tiêu cực.

Từ khoá: Đại sứ thương hiệu, người nổi tiếng, ý định mua, sự thu hút, sự tin cậy, chuyên môn, sự quen thuộc, sự phù hợp của người nổi tiếng với thương hiệu/sản phẩm, các thông tin tiêu cực.

\section{THE IMPACTS OF BRAND AMBASSADOR ON PURCHASE INTENTION AMONG CUSTOMERS IN HO CHI MINH CITY.}

\begin{abstract}
The purpose of this study is to investigate the factors of brand ambassador that influence the intention to purchase product among customers in Ho Chi Minh City. The research sample includes 210 responses of the participants aged 16 years old and above in Ho Chi Minh City. The survey was conducted via an online questionnaire. Data was analysied by SPSS 20 statistical software. The authors conduct linear regression analysis with purchase intention as the dependent variable and 6 independent variables including: attractiveness, trustworthiness, expertise, familarity, celebrity match-up congruence with the brand/product, negative publicity.
\end{abstract}

Keywords: brand ambassador, celebrity, purchase intention, attractiveness, trustworthiness, expertise, brand familarity, celebrity match-up congruence with the brand/product, negative publicity.

\section{GIỚI THIỆU}

Từ trước đến nay, khi bất kì một sản phẩm nào ra đời thì ngoài việc nghiên cứu và đầu tư cho chất lượng sản phẩm được tốt nhất thì việc quảng bá thương hiệu đến với người tiêu dùng cũng là một hoạt động không kém phần quan trọng (Todor, 2014). Hình ảnh doanh nghiệp, giá trị thương hiệu và uy tín là minh chứng cho sức cạnh tranh của doanh nghiệp. Khách hàng khi sử dụng thương hiệu cũng cảm thấy tự hào hơn (Kirmani, 2009). Chính vì thế mà hầu như các doanh nghiệp đều đã phải bỏ ra rất nhiều chi phí và công sức để đầu tư cho các hoạt động quảng bá thương hiệu. Trong sự cạnh tranh giành mức độ nhận diện thương hiệu tốt và chạy theo lợi nhuận, có không ít lần báo chí phanh phui những doanh nghiệp kinh doanh thiếu lương tâm và lừa dối khách hạng. Hậu quả là khiến người tiêu dùng ngày càng sinh ra tâm lý nghi ngờ. Hậu quả là họ sẽ nghĩ rằng quảng cáo luôn không bao giờ đúng với sự thật (Darke và Ritchie, 2007).. Bên cạnh đó xã hội ngày càng phát triển, mức sống ngày càng tăng lên thì cũng là lúc người tiêu dùng khó khăn hơn trong việc lựa chọn tin dùng một thương hiệu hay sản phẩm cụ thể. Để quảng bá cho thương hiệu, các nhà quản lý marketing phải đối mặt với một thử thách rằng họ phải luôn sáng tạo ra các chiến lược marketing khác biệt để thu hút và tạo được lòng tin cho khách hàng. Mỗi ngày người tiêu dùng đều tiếp cận với hàng loạt các quảng cáo khác nhau từ các nhãn hàng. Tạp chí marketing và truyền thông AdAge (2021) đã chỉ ra rằng khách hàng tiếp nhận khoảng 3.000 hình ảnh quảng cáo mỗi ngày, nhưng thực tế chỉ có 150 hình ảnh ảnh hưởng tới tiềm thức của khách hàng, trong số đó có 30 hình ảnh có thể tác động tới tâm thức của khách hàng (Giang và Dương, 2018). Lúc này, một chiến lược marketing hiệu quả chính là đầu tư vào xây dựng đại sứ thương hiệu. Đại sứ thương hiệu đã trở nên vô cùng phổ biến và cũng là một phần cần thiết trong 
chiến lược xây dựng thương hiệu của doanh nghiệp. Đặc biệt là những doanh nghiệp lớn. Để tạo được sự khác biệt, thu hút và có thể tiếp cận thêm nhiều đối tượng khách hàng, doanh nghiệp thường mời những ngôi sao đình đám làm đại sứ thương hiệu để quảng bá sản phẩm. Gương mặt ngôi sao gắn liền với hình ảnh sản phẩm giúp công chúng luôn nhớ tới nhãn hàng. Theo thống kê của công ty nghiên cứu thị trường Millward Brown, hơn $15 \%$ quảng cáo tại Mỹ có sự xuất hiện của người nổi tiếng, trong khi con số này tại Ấn Độ là $24 \%$ và Đài Loan lên tới $45 \%$ (Esangbedo, 2011). Sơn (2013) nhận định rằng sự xuất hiện của người nổi tiếng còn giúp cho một vài thương hiệu tăng doanh thu lên đến $20 \%$. Ở Việt Nam, các nhãn hàng mời người nổi tiếng làm đại diện cho thương hiệu của họ, điển hình như lần mà Sơn Tùng M-TP đại diện cho Biti's Hunter, chỉ với một đoạn ngắn xuất hiện đôi giày Biti’s Hunter trong video âm nhạc của Sơn Tùng M-TP nhưng đôi giày đã trở thành một chủ đề được bàn tán khá nhiều trong khoảng thời gian đó, được gọi là đôi giày "gây bão" và liên tục được tìm mua, giúp Biti's lội dòng ngoạn mục sau nhiều năm ít được nhắc đến. Đôi giày xuất hiện cùng Sơn Tùng có thể coi là cánh bướm mỏng manh mở màn cho hiệu ứng khủng khiếp sau đó của thương hiệu Biti's: Chỉ với 3 giây sản phẩm xuất hiện trong $\mathrm{MV}$ ca nhạc của Sơn Tùng M-TP, doanh số bán hàng của Biti's Hunter tăng lên $300 \%$ sau một tuần bán (VIỆT, 2021). Tuy nhiên, chi phí quảng cáo cho người nổi tiếng làm đại diện thương hiệu là vấn đề cần phải được cân nhắc kỹ lưỡng bởi doanh nghiệp. Thế nên, các nhà marketing cần phải có cơ sở để biết được những tác động thật sự giữa đại sứ thương hiệu với việc ra ý định mua của khách hàng, từ đó có thể đưa ra các quyết định đúng đắn trong việc đầu tư của họ. Bển cạnh đó, ở thời điểm hiện tại, một cách thức mới của chiến lược marketing truyền miệng là marketing thông qua người có sức ảnh hưởng đã trở nên phổ biến hơn rất nhiều. Theo thống kê của Digital Marketing Institute (2021), 49\% người tiêu dùng dựa vào đề xuất của các nhân vật có sức ảnh hưởng và $60 \%$ người tiêu dùng là giới trẻ tin theo lời khuyên của các nhân vật có sức ảnh hưởng hơn là người nổi tiếng. Theo Scott (2015), marketing dựa vào người ảnh hưởng là một chiến lược tiếp thị sử dụng ảnh hưởng của các cá nhân hoặc nhà lãnh đạo để thúc đẩy nhận thức về thương hiệu của người tiêu dùng và quyết định mua hàng của họ. Tuy nhiên, chiến lược marketing bằng đại sứ thương hiệu không còn hiệu quả? Để có thể một phần gợi ý cho các doanh nghiệp tại Việt Nam dễ dàng hơn trong việc chọn lựa ra các chiến lược xây dựng thương hiệu hiệu quả, có thể đưa thương hiệu của mình ra toàn cầu, phát triển nền kinh tế nước nhà, nhóm tác giả thực hiện nghiên cứu đề tài "Tác động của đại sứ thương hiệu lên ý định mua của khách hàng tại thành phố Hồ Chí Minh”.

\section{CƠ SỞ LÝ THUYÊT VÀ GIẢ THUYẾT NGHIÊN CƯU}

\subsection{Cơ sở lý thuyết}

\subsection{1 Đại sú thương hiệu}

Đại sứ thương hiệu hay có thể gọi là người đại diện thương hiệu là một chiến lược chiêu thị phổ biến để thu hút khách hàng. Đại sứ thương hiệu sẽ là đại diện cho mong muốn, nhu cầu và được người tiêu dùng dễ dàng chấp nhận. Họ sẽ là những linh vật, những chuyên gia lãnh đạo hay nhà lãnh đạo tôn giáo (Hafilah, Chaer, và Usman, 2019). Lý thuyết nền tảng về người có ảnh hưởng như đại sự thương hiệu đã xác định khả năng lãnh đạo quan điểm của những người có ảnh hưởng và mối quan hệ ngoài xã hội với những người có ảnh hưởng là hai cấu trúc trọng tâm ảnh hưởng đến ý định mua hàng của những người theo dõi (Farivar và cộng sự, 2021). Ngày nay, đại sứ thương hiệu thường sẽ là những người nổi tiếng như ca sĩ, diễn viên, người mấu, chuyên gia. Những người này được nhiều người yêu thích và có sức ảnh hưởng tới công chúng. Họ được các doanh nghiệp bổ nhiệm để doanh nghiệp sử dụng hình ảnh của họ làm đại diện cho nhãn hàng, thực hiện các hoạt động truyền thông nhằm quảng bá thương hiệu như: xuất hiện trong buổi họp báo ra mắt sản phẩm, trong các chuỗi quảng cáo của nhãn hàng hay đăng tải bài viết liên quan đến nhãn hàng mà họ đại diện trên các trang mạng xã hội cá nhân. Các thương hiệu được quảng cáo thường nhận được sự đại diện bởi nhiều nhân vật nổi tiếng của công chúng. Điều này được ước tính rằng có khoảng $1 / 6$ số quảng cáo trền toàn thế giới có những người nổi tiểng (A.Shimp và Andrews, 2012). Việc bổ nhiệm đại sứ thương hiệu còn là một chiến lược sáng tạo nhằm duy trì và tăng sự quan tâm cũng như định vị hình ảnh của thương hiệu, truyền tải thông điệp rộng rãi đến người tiêu dùng.

\subsection{2 Ý định mua hàng}

Ý định hành vi (behavioral intention), hay gọi tắt là ý định (intention) là một khái niệm rất quan trọng trong lĩnh vực kinh doanh nói riêng và những lĩnh vực khác nói chung (Giao và Trà, 2020).Ý định tham gia vào một hành vi càng mạnh thì khả năng thực hiện hành vi đó càng cao (Ajzen, 1991). Ý định là một yếu tố 
dùng để đánh giá khả năng thực hiện hành vi trong tương lai (Thắng và Độ, 2016). Ý định mua là một loại của việc ra quyết định mua hàng mà khi đó người tiêu dùng nghiên cứu lý do để mua một thương hiệu cụ thể (Shah và cộng sự, 2012). Ý định mua hàng thường liên quan đến hành vi, nhận thức và thái độ của người tiêu dùng. Ý định mua có thể bị thay đổi dưới ảnh hưởng của giá cả, chất lượng và giá trị mà người tiêu dùng cảm nhận được. Kotler và cộng sự (2009) đã đề xuất sáu giai đoạn trước khi quyết định mua sản phẩm, đó là: nhận thức, tìm kiếm thông tin, quan tâm đến sản phẩm, ưa thích sản phẩm, thuyết phục và quyết định mua. Ngoài ra, người tiêu dùng còn bị ảnh hưởng bởi các động cơ bên trong hoặc bên ngoài trong quá trình mua hàng. Khách hàng luôn có suy nghĩ rằng mua hàng với chi phí thấp, bao bì đơn giản và sản phẩm ít tên tuổi là rủi ro cao vì chất lượng của những sản phẩm này không đáng tin cậy (Gogoi, 2013). Hiểu rõ các yếu tố ảnh hưởng đến ý định mua hàng của người tiêu dùng là vấn đề quan trọng trong việc duy trì và gia tăng số lượng khách hàng.

\subsubsection{Lý thuyết hành vi người tiêu dùng}

Lý thuyết Hành động hợp lý (Fishbein và Ajzen, 1975) và lý thuyết Hành vi dự định (Ajzen, 1991) là những lý thuyết tiêu biểu bắt nguồn từ nghiên cứu tâm lý xã hội và được ứng dụng rộng rãi trong bối cảnh marketing hiện tại, đặc biệt là nghiên cứu về hành vi của người tiêu dùng. Theo Nguyễn và Lê (2021), hành vi của một người sẽ được quyết định bởi ý định thực hiện hành vi của người đó và ý định này chịu ảnh hưởng bởi hai yếu tố: thái độ đối với hành vi và chuẩn chủ quan. Theo Nguyễn (2021) Hành vi người tiêu dùng là một thuật ngữ chỉ tất cả các hoạt động liên quan đến việc mua hàng, sử dụng và ngưng sử dụng các sản phẩm hàng hóa, dịch vụ của người tiêu dùng. Nó bao gồm các phản ứng, thái độ về cảm xúc, tinh thần và hành vi tiêu dùng của người tiêu dùng đối với các sản phẩm hàng hóa, dịch vụ. Cũng theo (Nguyễn, 2021), các yếu tố ảnh hưởng đến hành vi người tiêu dùng đầu tiên là yếu tố văn hóa xã hội, yếu tố này ảnh hưởng nhiều nhất đối với hành vi người tiêu dùng, ở mỗi một nơi sẽ có nền văn hóa khác nhau, giai tầng xã hội khác nhau dẫn tới hành vi tiêu dùng khác nhau.

\subsubsection{Lý thuyết về tác động của đại súu thuong hiệu lên hành vi nguời tiêu dùng}

Trong bối cảnh marketing hiện đại, các thương hiệu đã sử dụng những người có ảnh hưởng trên mạng xã hội làm người xác nhận sản phẩm và dịch vụ của họ cũng như làm đại sứ thương hiệu. Những người có ảnh hưởng kỹ thuật số kết nối người tiêu dùng và thương hiệu, củng cố mối quan hệ của họ và cho phép thương hiệu đạt được mục tiêu của họ theo cách tự nhiên hơn để tác động đến quá trình mua hàng của người tiêu dùng (Antunes, 2021). Utami và công sự (2020) chứng minh rằng đại sứ thương hiệu và chất lượng của truyền miệng điện tử (e-WOM) có ảnh hưởng tích cực đến ý định mua hàng của người tiêu dùng. Trong bối cảnh nghiên cứu tại Việt Nam, Huynh (2020) xác định rằng người có ảnh hưởng qua các kênh mạng xã hội như đại sứ thương hiệu có ảnh hưởng trong suốt ba giai đoạn đầu tiên của quá trình ra quyết định của người tiêu dùng. Các người ảnh hưởng này đạt được ảnh hưởng của họ thông qua việc tạo ra các nội dung hữu ích và phù hợp với người tiêu dùng. Tuy nhiên, khi các người ảnh hưởng này phát triển về quy mô và thu hút nhiều lời đề nghị tài trợ hơn, tính xác thực của các đề xuất sản phẩm của họ trở nên đáng ngờ.

\subsection{Giả thuyết nghiên cứu}

\subsubsection{Sư tin cây}

Các thành phần chính của sự tin cậy là có thể tin cậy, có thể dựa theo, trung thực, chân thành và đáng tin cậy. Phần lớn người trẻ tuổi thường dựa theo những lời khuyên hơn là người lớn tuổi vì họ muốn đi theo xu hướng của những người nổi tiếng (Pandey, 2011). Bất kỳ người nào có thể gây ảnh hưởng tích cực hoặc tiêu cực đến thái độ của người tiêu dùng được gọi là nhóm người tham khảo và những người nổi tiếng là nhóm người tham khảo đáng tin cậy nhất (Escalas và Bettman, 2005). Phụ nữ tin tưởng nhiều hơn vào những người nổi tiếng phụ nữ và nam giới vào những người nổi tiếng là nam giới (Sliburyte, 2009). Các công ty cũng có thể tận dụng người đại diện có sự tín nhiệm cao, những người mà họ có danh tiếng, độ phổ biến, hình ảnh công chúng tốt và độ tín cậy cao (Song, Chaipoopiratana và cộng sự, 2008). Người tiêu dùng thường coi những người nổi tiếng là nguồn thông tin giao tiếp đáng tin cậy (Goldsmith và cộng sự, 2000). Osei-Frimpong và cộng sự (2019) đã chứng minh rằng sự đáng tin cậy của đại sứ thương hiệu có ảnh hưởng cùng chiều đến ý định mua của người tiêu dùng. Weismueller và cộng sự (2020) cũng cho rằng sự đáng tin cậy của đại sứ thương hiệu có ảnh hưởng tích cực đến ý định mua của khách hàng. Theo Utami và cộng sự (2020), xem các đánh giá trực tuyến đáng tin cậy từ đại sứ thương hiệu sẽ hữu ích cho người tiêu dùng 
trong việc xây dựng niềm tin của họ vào ý định mua hàng. Từ các nhận định trên, giả thuyết $\mathrm{H} 1$ được đưa ra như sau:

Giả thuyết H1: Sự tin câyy của đại sú thuơng hiệu có tác động cùng chiều (+) đến ý định mua của nguời tiêu dùng tại TP. Hồ Chí Minh.

\subsubsection{Sur thu hút}

Truyền thống quan niệm rằng ngoại hình đẹp sẽ có ý nghĩa đối với mọi người khi mua một thứ gì đó (Reingen và Kernan, 1993). Những diện mạo của quảng cáo đẹp sẽ tạo ra cảm giác hấp dẫn và tăng thái độ tích cực, thu hút sự chú ý mua hàng của người xem (Clow, James và cộng sự 2006). Các công trình của nhiều nhà nghiên cứu cũng chứng minh rằng ngoại hình và sự hấp dẫn về thể chất là một yếu tố đặc biệt để kiểm tra hiệu quả của người nổi tiếng (Baker, Churchill và cộng sự, 1977; Joseph 1982; Kahle và Homer, 1985; Winham và Hampl, 2008). Nhưng theo Bower và Landreth (2001), những người mẫu có ngoại hình hấp dẫn đôi khi cũng không hiệu quả trong quảng cáo. Sức hấp dẫn có nhiều chiều và rất khó xác định, không thể xác định được mức độ hâp dẫn chỉ qua một chiều duy nhất (Caballero và Solomon, 1984). Việc tạo nên sự thu hút không chỉ ở vẻ đẹp và ngoại hình mà các thuộc tính phi vật lý cũng đóng một vai trò quan trọng trong mức độ thu hút của người nổi tiếng như thành tích, sự khéo léo, trí thông minh (Kamins, 1990; Sliburyte, 2009) sự liên quan đến nhóm người tiêu dùng (Deshpandé và Stayman, 1994). OseiFrimpong và cộng sự (2019) đã chứng minh rằng sự thu hút của đại sứ thương hiệu có ảnh hưởng tích cực đến ý định mua của người tiêu dùng. Weismueller và cộng sự (2020) cũng cho rằng sự thu hút của đại sứ thương hiệu có ảnh hưởng tích cực đến ý định mua của khách hàng Utami và cộng sự (2020) cũng xác nhận sự thu hút của đại sứ thương hiệu có tác động cùng chiều đến ý định mua của người tiêu dùng. Từ các nhận định trên, giả thuyết $\mathrm{H} 2$ được đưa ra như sau:

Giả thuyết H2: Sự thu hút của đại sút thưong hiệu có tác động cùng chiều (+) đến ý định mua của ngườ tiêu dùng tại Tp. Hồ Chi Minh.

\subsubsection{Chuyên môn}

Thuật ngữ "chuyên môn" được định nghĩa là mức độ mà một người giao tiếp được coi là nguồn cung cấp các khẳng định hợp lệ (Hovland và cộng sự, 1953). Để thuyết phục người tiếp nhận thông tin, kiến thức chuyên môn của người đại diện có tác dụng đáng khích lệ đối với người tiếp nhận (Ohanian, 1990). Belch và Belch (1994) cho rằng người tiếp nhận thông tin có niềm tin mạnh mẽ vào người thực sự có kiến thức chuyên môn trong lĩnh vực. Người đại diện với kiến thức và kỹ năng cao có tác động khuyển khích mạnh mẽ so với những người đại diện có chuyên môn thấp (Ohanian, 1990). Chuyên môn của người nổi tiếng là một trong những lý do để tìm ra ảnh hưởng của nó đối với người tiếp nhận thông tin (Amos, Holmes và Strutton, 2008). Người tiêu dùng sẽ đánh giá chuyên môn của người đại diện thương hiệu qua giá trị thông tin nhận thức được. Weismueller và cộng sự (2020) xác nhận chuyên môn của đại sứ thương hiệu có ảnh hưởng tích cực đến ý định mua của khách hàng. Theo quan điểm này, Fernández và Castillo (2021) xác định rằng giá trị thông tin nhận thức được trong quá trình gây ảnh hưởng có thể khiến những người theo dõi thể hiện ý định hành vi đối với các thương hiệu được những người có ảnh hưởng xác nhận. Từ các nhận định trên, giả thuyết $\mathrm{H} 3$ được đưa ra như sau:

Giả thuyết H3: Chuyên môn của đại sứ thưong hiệu có tác động cùng chiều (+) đến ý định mua của nguời tiêu dùng tại TP. Hồ Chí Minh.

\subsubsection{Sư quen thuộc}

Sự quen thuộc với thương hiệu (Brand Familiarity) phản ánh mức độ trải nghiệm trực tiếp và gián tiếp của người tiêu dùng với một thương hiệu (Alba và Hutchinson, 1987; Kent và Allen 1994). Sự quen thuộc với thương hiệu nắm bắt được cấu trúc kiến thức thương hiệu của những người kết luận, tức là những xã hội thương hiệu tồn tại trong trí nhớ của người tiêu dùng. Sự quen thuộc có nghĩa là cảm giác đồng điệu bởi ý nghĩa về mặt cảm xúc và sự giao tiếp với người nổi tiếng. Sự quen thuộc của người nổi tiếng sẽ có nhiều tác động tích cực hơn khi chính khách hàng tìm thấy được rằng bản thân họ có sự đồng điều với người nổi tiếng (Fernández và Castillo, 2021). Điều này gọi là hiệu ứng tiếp xúc đơn thuần (mere-exposure effect) (Wills, 2021). Khi khách hàng có khoảng thời gian tương tác dài và những tương tác gần với người nổi tiếng thì sự quen thuộc sẽ có tác động đáng kể tới thái độ của khách hàng đối với thương hiệu (Ha và Lam, 2016). Utami và cộng sự (2020) đã xác nhận rằng sự quen thuộc của đại sứ thương hiệu có tác động cùng chiều đến ý định mua của người tiêu dùng. Ngoài ra, Osei-Frimpong và cộng sự (2019) cũng chỉ ra rằng người 
chứng thực người nổi tiếng có các thuộc tính như sự hấp dẫn, đáng tin cậy và quen thuộc có ảnh hưởng tích cực đến nhận thức của người tiêu dùng về chất lượng, ý định mua hàng và lòng trung thành với thương hiệu.Từ các nhận định trên, giả thuyết $\mathrm{H} 4$ được đưa ra như sau:

Giả thuyết H4: Sự quen thuộc của đại sứ thương hiệu có tác động cùng chiều (+) đến ý định mua của người tiêu dùng tại TP. Hồ Chí Minh.

\subsubsection{Sự phù hợp giũua đại sú thưong hiệu với thưong hiệ/sản phẩm}

Sự phù hợp giữa người nổi tiếng với thương hiệu/sản phẩm có một vai trò quan trọng trong marketing hiện đại. Erdogan (1999) cho rằng phải có sự cân bằng hoàn hảo giữa các thuộc tính của thương hiệu và hành vi hoặc tính cách của người nổi tiếng đó đại diện. Mức độ phù hợp hiệu quả có thể được đo lường bằng mức độ phù hợp giữa người có ảnh hưởng và người ủng hộ. Kalu (2019) đã khẳng định sự phù hợp giữ đại sứ thương hiệu trên mạng xã hội ảnh hưởng đến ý định mua của khách hàng thuộc thế hệ millennial. Khi một sản phẩm được quảng cáo bởi một người nổi tiếng với hình ảnh phù hợp có liên quan nhiều đến sản phẩm, thì sự tin tưởng vào quảng cáo và người nổi tiếng sẽ cao hơn so với hình ảnh sản phẩm được quảng bá bởi một người ít nổi tiếng hơn, ít liên quan hơn (Ha và Lam, 2016). Trong bối cảnh có sự phù hợp giữa đại sứ thương hiệu và sản phẩm, Farivar và cộng sự (2021) đã xác nhận tác động tích cực của việc dẫn dắt ý kiến đối với ý định mua hàng của người theo dõi. Từ các nhận định trên, giả thuyết H5 được đưa ra như sau:

Giả thuyết H5: Sự phù hợp của đại sứ thuơng hiệu với thuơng hiệu/sản phẩm tác động cùng chiều (+) đến ý định mua của người tiêu dùng tại TP. Hồ Chí Minh.

\subsubsection{Các thông tin tiêu cưc}

Việc sử dụng đại sứ thương hiệu luôn tốn rất nhiều chi phí của doanh nghiệp. Chi phí đó không chỉ là số tiền mà họ bỏ ra để trả cho người nổi tiếng mà còn là chi phí khi xuất hiện những rủi ro từ đại sứ thương hiệu, những rủi ro đó chính là các thông tin tiêu cực của đại sứ thương hiệu. Knott và James (2004) đã khẳng định việc sử dụng những người nổi tiếng dính vào những thông tin tiêu cực có thể gây ra hậu quả nghiêm trọng về hiệu suất hoặc sự chấp nhận thương hiệu. Tương tự, Thwaties và cộng sự (2012) cũng phát hiện rằng các thông tin tiêu cực từ đại sứ thương hiệu ảnh hưởng đến sức hấp dẫn và độ tin cậy của họ. Prameswara và Sjabadhyni (2018) đã khẳng định tác động tiêu cực của người nổi tiếng và sự nhận diện thương hiệu lên ý định mua của người tiêu dùng. Tuy nhiên, Osei-Frimpong và cộng sự (2019) lại chứng minh những thông tin truyền thông tiêu cực không ảnh hưởng đến ý định mua của người tiêu dùng đối với thương hiệu mà người đó đại diện. Hafilah và cộng sự (2019) cũng ủng hộ quan điểm đại sứ thương hiệu không ảnh hưởng nhiều đến quyết định mua của người tiêu dùng. Từ các quan điểm trên, giả thuyết H6 được đưa ra như sau:

Giả thuyết H6: Các thông tin tiêu cực của nhũng người đại diện thương hiệu làm suy yếu (-) tác động của họ đối với ý định mua hàng của người tiêu dùng tại TP. Hồ Chí Minh.

Từ tổng quan nghiên cứu trên, mô hình nghiên cứu được đề xuất:

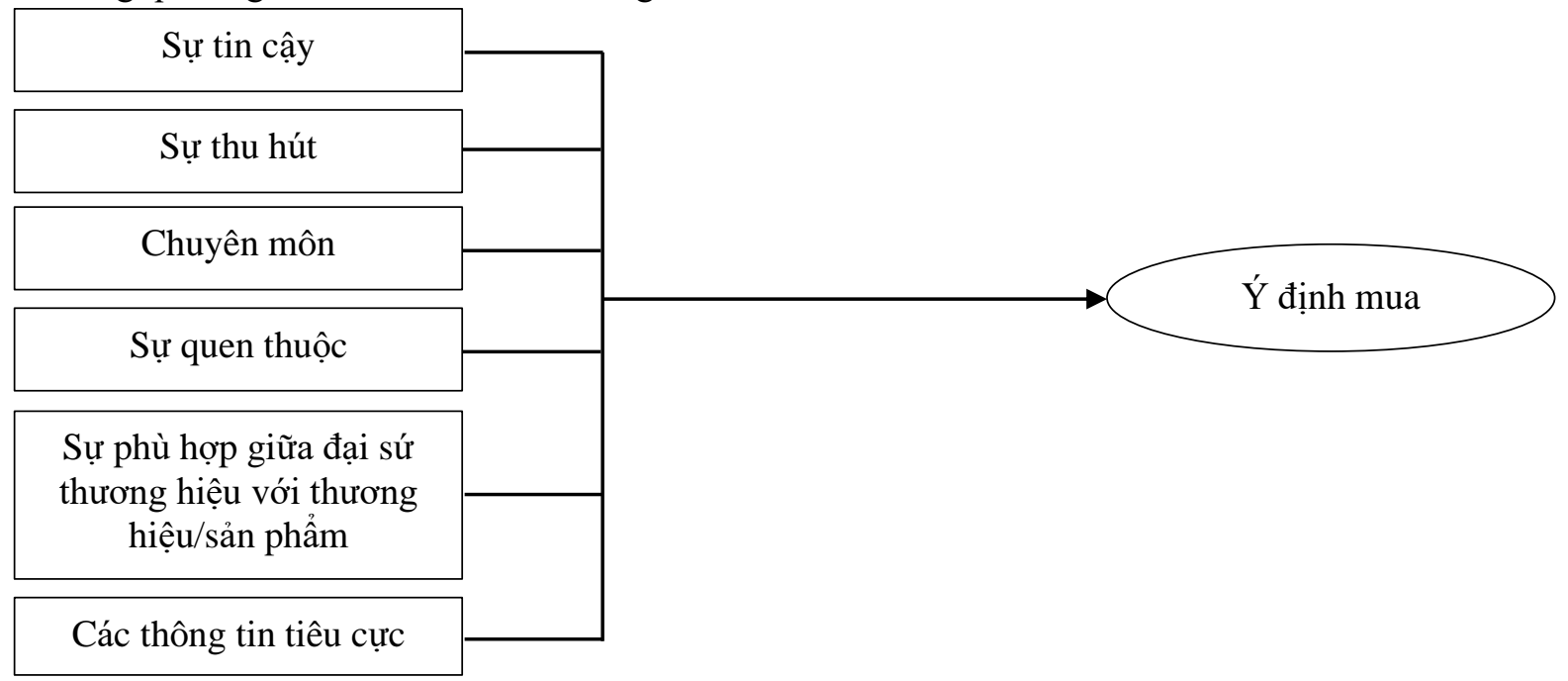

Hình 1. Mô hình nghiên cứu 


\section{PHƯƠNG PHÁP NGHIÊN CÚU}

Nghiên cứu được thực hiện bằng phương pháp nghiên cứu định lượng. Trong đó, phương pháp nghiên cứu định lượng sử dụng phương pháp điều tra bằng bảng câu hỏi. Nghiên cứu được tiển hành qua 03 giai đoạn chính. Giai đoạn đầu là giai đoạn thiết kế thang đo nháp và thực hiện khảo sát nghiên cứu định lượng sơ bộ. Ở giai đoạn này tác giả tiến hành xây dựng thang đo nháp dựa vào sự tham khảo các nghiên cứu liên quan trong và ngoài nước. Sau đó, nhóm tác giả tiến hành nghiên cứu sơ bộ với đối tượng khảo sát là đang sống và làm việc tại $\mathrm{TP}$. Hồ Chí Minh. Cách thức thu thập dữ liệu là khảo sát trực tiếp với cỡ mẫu $\mathrm{n}=50$ nhằm kiểm định sơ bộ độ tin cậy của thang đo các thành phần trong khái niệm nghiên cứu thông qua hệ số Cronbach's Alpha. Kết quả của giai đoạn này là đưa ra thang đo và bảng câu hỏi chính thức. Giai đoạn thứ hai là tiến hành nghiên cứu chính thức. Được thực hiện thông qua nghiên cứu định lượng với đối tượng khảo sát là các cá nhân đang sống và làm việc tại $\mathrm{TP}$. Hồ Chí Minh bằng bảng khảo sát nhằm kiểm định thang đo và mô hình nghiên cứu lý thuyết. Giai đoạn cuối cùng giai đoạn xử lí dữ liệu và đưa ra kết luận. Việc thu thập dữ liệu được thực hiện bằng cách khảo sát trực tuyến (gửi link Google form qua mạng). Các dữ liệu thô sau khi thu về sẽ được lọc để loại ra các phản hồi bị lỗi hay thiếu thông tin. Bảng câu hỏi sử dụng thang đo Likert 5 cho 7 nhân tố trong mô hình nghiên cứu với mức độ với thứ tự tăng dần từ 01 (hoàn toàn không đồng ý), 02 (không đồng ý), 03 (không ý kiến), 04 (đồng ý) và 05 (hoàn toàn đồng ý). Trong nghiên cứu định lượng, để kiểm định lý thuyết khoa học, chọn mẫu là một trong những khâu quyết định chất lượng của kết quả nghiên cứu (Thọ, 2013). Theo Hoàng và Chu (2008), thông thường số quan sát (cỡ mẫu) ít nhất phải bằng 4 hay 5 lần số biến trong phân tích nhân tố EFA. Trong thang đo của tác giả có tổng cộng 31 biến quan sát, vì vậy cỡ mẫu tối thiểu tức phiếu khảo sát tối thiểu cần để thực nghiên nghiên cứu chính thức là: $\mathrm{n}=31 * 5=155$ (phiếu khảo sát).Nhưng để đảm bảo an toàn và độ tin cậy của dữ liệu khi phân tích, tác giả lựa chọn cỡ mẫu lớn hơn 155 người và vì trong quá trình nghiên cứu, tác giả có thời gian và tài chính hạn hẹp, thế nên để chọn mẫu, tác giả sử dụng phương pháp phi xác suất, lấy mẫu thuận tiện. Đối tượng khảo sát là người tiêu dùng đang sống/học tập/làm việc tại Thành phố Hồ Chí Minh. Nhóm tác giả thu về được 260 phiếu khảo sát với 50 phiếu không hợp lệ (đáp viên trả lời không tập trung hay qua loa) và 210 phiếu hợp lệ (lớn hơn cỡ mẫu tối thiểu) được dùng cho phân tích. Tác giả lựa chọn phương pháp chọn mẫu thuận tiện phi xác suất với kích thước mẫu là 210 người từ 16 tuổi trở lên đang sinh sống và làm việc tại khu vực Tp. Hồ Chí Minh.Sau đó tác giả tiến hành phân tích bằng phần mềm SPSS 20 với các phương pháp: thống kê mô tả, kiểm định độ tin cậy bằng Cronbach's Alpha, phân tích nhân tố khám phá EFA, phân tích tương quan, hồi quy tuyến tính, kiếm định T-Test, ANOVA.

\begin{tabular}{|l|l|l|l|l|}
\hline STT & Nhân tố & Số biến quan sát & Mã hóa & Nguồn tham khảo \\
\hline 1 & Sự tin cậy & 5 & TC & Giang và Dương (2018) \\
\hline 2 & Sự thu hút & 5 & TH & Giang và Dương (2018) \\
\hline 3 & Chuyên môn & 4 & CM & Giang và Dương (2018) \\
\hline 4 & $\begin{array}{l}\text { Sự quen thuộc } \\
\text { Sự phù hợp giữa đại sứ thương } \\
\text { hiệu/sản phâm }\end{array}$ & 4 & QT & $\begin{array}{l}\text { Osei-frimpong và cộng sự } \\
\text { (2019) }\end{array}$ \\
\hline 5 & $\begin{array}{l}\text { Các thông tin tiêu cực } \\
7\end{array}$ & $\begin{array}{l}\text { Ý định mua } \\
\text { Ha và Lam (2017) }\end{array}$ & PH & Haingong và cộng sự \\
\hline
\end{tabular}

Hình 2. Các nhân tố trong mô hình nghiên cứu. 


\section{KẾT QUẢ NGHIÊN CÚU}

\section{1. Đặc điểm mẫu nghiên cứu}

Bảng 1. Đặc điểm mẫu nghiên cứu $(\mathrm{n}=210)$

\begin{tabular}{|l|l|l|}
\hline \multirow{4}{*}{ Giới tính } & \multicolumn{1}{|c|}{ Ty lẹ (\%) } \\
\hline \multirow{4}{*}{ Độ tuổi } & Nam & 42.9 \\
\cline { 2 - 3 } & Nữ & 57.1 \\
\hline \multirow{4}{*}{ Nghề nghiệp } & Từ $16-22$ tuổi & 42.4 \\
\cline { 2 - 3 } & Từ $23-28$ tuổi & 39.0 \\
\cline { 2 - 3 } & Từ $29-35$ tuổi & 15.2 \\
\cline { 2 - 3 } & Trên 35 tuổi & 3.3 \\
\hline \multirow{5}{*}{ Thu nhập } & Học sinh - sinh viên & 34.8 \\
\cline { 2 - 3 } & Nhân viên văn phòng & 42.9 \\
\cline { 2 - 3 } & Người lao động phổ thông & 15.2 \\
\cline { 2 - 3 } & Cán bộ - viên chức & 3.3 \\
\cline { 2 - 3 } & Khác & 3.8 \\
\hline & Dưới 5 triệu đồng & 24.8 \\
\cline { 2 - 3 } & Từ 5 đến dưới 10 triệu đồng & 34.8 \\
\cline { 2 - 3 } & Từ 10 đến dưới 20 triệu đồng & 31.4 \\
\cline { 2 - 3 } & Trên 20 triệu đồng & 9.0 \\
\hline
\end{tabular}

\section{2 Đánh giá độ tin cậy của thang đo bằng Cronbach's Alpha}

Bảng 2. Đánh giá độ tin cậy của thang đo bằng Cronbach's Alpha

\begin{tabular}{|l|l|l|l|}
\hline Yếu tố & $\begin{array}{l}\text { Số biến quan } \\
\text { sát }\end{array}$ & Hệ số Cronbach's Alpha & $\begin{array}{l}\text { Hệ số tương quan biến } \\
\text { tổng nhỏ nhẩt }\end{array}$ \\
\hline Sự tin cậy & 5 & 0.928 & 0.702 \\
\hline Sự thu hút & 5 & 0.922 & 0.728 \\
\hline Chuyên môn & 4 & 0.886 & 0.685 \\
\hline Sự quen thuộc & 6 & 0.943 & 0.754 \\
\hline $\begin{array}{l}\text { Sự phù hợp của đại sứ thương hiệu với } \\
\text { thương hiệu/sản phẩm }\end{array}$ & 4 & 0.925 & 0.789 \\
\hline Các thông tin tiêu cực & 3 & 0.889 & 0.744 \\
\hline Ý định mua & 3 & 0.883 & 0.652 \\
\hline
\end{tabular}

Dựa vào kết quả kiểm định độ tin cậy của thang đo có thể thấy, hệ số Cronbach's Alpha đều lớn hơn 0.6 và hệ số tương quan biến tổng đều lớn hơn 0.3 . Qua đó, cho thấy thang đo các khái niệm đều đảm bảo yêu cầu về độ tin cậy.

\subsection{Phân tích nhân tố khám phá EFA}

Sau khi kiểm định Cronbach's Alpha, mô hình nghiên cứu có 6 nhóm nhân tố với 27 biến quan sát ảnh hưởng đến ý định mua. Sau khi khảo sát, dùng phương pháp phân tích nhân tố khám phá EFA để đo lường sự tương thích của mẫu khảo sát. Các con số trong bảng Rotated Component Matrix (ma trận xoay) thể hiện các trọng số nhân tố hay hệ số tải nhân tố (Factor loading) lớn nhất của mỗi biến quan sát. Người thực hiện đề tài chạy 1 lần ra kết quả phân thành 6 nhóm biến. Dưới đây là kết quả phân tích nhân tố. 
TẠI THÀNH PHỐ HỒ CHÍ MINH

Bảng 3. Kết quả phân tích EFA cho 6 biến độc lập

\begin{tabular}{|c|c|c|c|c|c|c|}
\hline \multirow{2}{*}{ Mã hóa } & \multicolumn{6}{|c|}{ Nhân tố } \\
\hline & 1 & 2 & 3 & 4 & 5 & 6 \\
\hline QT6 & .895 & & & & & \\
\hline QT4 & .879 & & & & & \\
\hline QT3 & .865 & & & & & \\
\hline QT5 & .858 & & & & & \\
\hline QT1 & .827 & & & & & \\
\hline QT2 & .793 & & & & & \\
\hline TC5 & & .925 & & & & \\
\hline $\mathrm{TC} 4$ & & .904 & & & & \\
\hline $\mathrm{TC} 1$ & & .861 & & & & \\
\hline $\mathrm{TC} 2$ & & .819 & & & & \\
\hline TC3 & & .761 & & & & \\
\hline TH5 & & & .925 & & & \\
\hline TH4 & & & .917 & & & \\
\hline TH1 & & & .847 & & & \\
\hline TH3 & & & .834 & & & \\
\hline TH2 & & & .816 & & & \\
\hline $\mathrm{CM} 4$ & & & & .877 & & \\
\hline CM1 & & & & .873 & & \\
\hline CM3 & & & & .806 & & \\
\hline $\mathrm{CM} 2$ & & & & .762 & & \\
\hline PH1 & & & & & .844 & \\
\hline PH4 & & & & & .838 & \\
\hline $\mathrm{PH} 3$ & & & & & .753 & \\
\hline $\mathrm{PH} 2$ & & & & & .752 & \\
\hline TTTC1 & & & & & & .884 \\
\hline TTTC3 & & & & & & .828 \\
\hline TTTC2 & & & & & & .810 \\
\hline Hệ số KMO & 0.850 & & & & & \\
\hline Sig & 0.000 & & & & & \\
\hline Eigenvalues & 1.326 & & & & & \\
\hline Tổng phương sai trích & 79.00 & & & & & \\
\hline
\end{tabular}

Trong bảng 3, ta có hệ số KMO của kiểm định phù hợp với mô hình đạt $0.850(0.5<\mathrm{KMO}<1)$, chứng tỏ các biến đưa vào phân tích nhân tố là có ý nghĩa và mô hình phân tích phù hợp với các giả thiết đã đề ra (Hair, 2006).Tiếp theo kiểm định tương quan biến có $\mathrm{Sig}=0<0.05$. Chứng tỏ giả thiết các biến không tương quan với nhau bị bác bỏ và kết luận các biến có tương quan với nhau, phù hợp với việc phân tích nhân tố. Qua hai kiểm định trên, ta thấy việc phân tích nhân tố đối với các yếu tố ảnh hưởng tới ý định mua là phù hợp. Tiêu chuẩn tiếp theo để xác định các biến phù hợp để đưa vào phân tích nhân tố là xác định hệ số tải nhân tố (Factor Loading) của các biến, ta có các biến đều có hệ số tải nhân tố lớn hơn 0.5 nghĩa là các nhân tố được xem là có ý nghĩa thực tiễn. Eigenvalues $=1.326>1$ đại diện cho phần biến thiên được giải thích bởi mỗi nhân tố, thì nhân tố rút ra có ý nghĩa tóm tắt thông tin tốt nhất (Hair, 2006).. Ta có tổng 
phương sai trích Total Variance Explained $=79.003 \%>50 \%$, như vậy $79.003 \%$ sự biến thiên của dữ liệu được giải thích bởi 6 nhân tố. Như vậy mô hình cuối cùng còn lại 27 biến quan sát được chia vào 6 nhóm như bảng 3 phí trên.

\subsection{Phân tích tương quan và hồi quy đa biến}

\subsubsection{Phân tích tuoong quan Pearson}

Bảng 4. Ma trận hệ số tương quan Pearson

\begin{tabular}{|l|l|l|l|l|l|l|l|}
\hline & YD & TC & TH & CM & QT & PH & TTTC \\
\hline YD & 1 & & & & & & \\
\hline TC & 0.506 & 1 & & & & & \\
\hline TH & 0.254 & 0.107 & 1 & & & & \\
\hline CM & 0.527 & 0.230 & 0.114 & 1 & & & \\
\hline QT & 0.540 & 0.257 & 0.153 & 0.314 & 1 & & \\
\hline PH & 0.778 & 0.371 & 0.211 & 0.480 & 0.492 & 1 & \\
\hline TTTC & -0.525 & -0.399 & -0.086 & -0.287 & -0.329 & -0.482 & 1 \\
\hline
\end{tabular}

Kết quả phân tích tương quan Pearson cho thấy cho thấy giữa các biến độc lập (TC, TH, CM, QT, PH, TTT) và biến phụ thuộc $(\mathrm{YD})$ có Sig. $=0.00<0.05$ đủ điều kiện để tiến hành chạy phân tích hồi quy (Hair, 2006). Các biến độc lập đều có tương quan tới ý định mua, riêng biến PH có tác động mạnh mẽ nhất. Khi đánh giá chung về sự phù hợp của đại sứ thương hiệu với thương hiệu/sản phẩm, sự tin cậy, sự thu hút, chuyên môn, sự quen thuộc và các thông tin tiêu cực càng cao thì đánh giá chung về tổng thể ý định mua cũng cao. Và ngược lại, khi khách hàng không hài lòng về bất kỳ yếu tố nào trong bảy yếu tố trên thì đánh giá chung sẽ vì thế mà giảm xuống.

\subsubsection{Phân tích hồi quy đa biến}

Bảng 5. Kết quả phân tích hồi quy đa biến

\begin{tabular}{|c|c|c|c|c|c|}
\hline Mô hình & B & Beta & $\mathbf{t}$ & Sig. & VIF \\
\hline Hằng số & 0.885 & & 3.778 & 0.000 & \\
\hline TC & 0.157 & 0.199 & 4.771 & 0.000 & 1.260 \\
\hline TH & 0.061 & 0.079 & 2.084 & 0.038 & 1.052 \\
\hline CM & 0.142 & 0.162 & 3.798 & 0.000 & 1.320 \\
\hline QT & 0.111 & 0.153 & 3.544 & 0.000 & 1.359 \\
\hline PH & 0.343 & 0.482 & 9.555 & 0.000 & 1.847 \\
\hline TTTC & -0.063 & -0.110 & -2.488 & 0.014 & 1.424 \\
\hline $\mathbf{R}$ & \multicolumn{5}{|l|}{0.849} \\
\hline $\mathbf{R}^{2}$ & \multicolumn{5}{|l|}{0.721} \\
\hline $\mathbf{R}^{2}$ hiệu chỉnh & \multicolumn{5}{|l|}{0.713} \\
\hline Durbin-Watson & \multicolumn{5}{|l|}{2.282} \\
\hline F (87.385) & \multicolumn{5}{|c|}{ Sig. $=0.000$} \\
\hline
\end{tabular}

Dựa theo kết quả phân tích hồi quy, ta có giá trị $\mathrm{R}^{2}$ hiệu chỉnh là 0.713 , nghĩa là 6 biến độc lập đưa vào ảnh hưởng $71.3 \%$ sự thay đổi của biến phụ thuộc (YD), còn lại $28,7 \%$ là do các biến ngoài mô hình và sai số ngẫu nhiên. Và $71.3 \%>50 \%$ nên nghiên cứu được đánh giá tốt (Hoàng và Chu, 2008). Kiểm định hiện tượng tự tương quan của phần dư: Hệ số $0<$ Durbin Watson $=2.282<4$, do đó không xuất hiện hiện tượng tương quan chuỗi bậc nhất. Mức độ phù hợp của mô hình (Phân tích phương sai ANOVA): Độ tin cậy 95\%, Sig. $<0.05$, chứng tỏ mô hình lý thuyết phù hợp với thực tế. Các biến độc lập có tương quan tuyến tính với biến phụ thuộc trong mô hình. Giá trị Sig kiểm định của 6 biến độc lập đều $<0.05$ nghĩa là các biến độc lập tương quan và có ý nghĩa với biến phụ thuộc $\mathrm{Y}$, với độ tin cậy $95 \%$. Do vậy các hệ số này phù hợp để đưa 
vào phương trình hồi quy (Hair, 2006). Tiếp đến ta có giá trị VIF kiểm tra hiện tượng đa cộng tuyến. Thông thường VIF < 10 sẽ không có hiện tượng đa cộng tuyến, tuy nhiên theo một số nghiên cứu trước đây, với bài nghiên cứu có mô hình và bảng câu hỏi sử dụng thang đo Likert thì VIF < 2 sẽ không có hiện tượng đa cộng tuyến (Hair, 2014). Hệ số hồi quy chuẩn hóa (Beta) cho thấy mức độ tác động của các biến độc lập đến biến ý định mua và được sắp xếp theo thứ tự sau: Sự phù hợp giữa đại sứ thương hiệu với thương hiệu và sản phẩm $(\beta=0.482)$, Sự tin cậy $(\beta=0.199)$, Chuyên môn $(\beta=0.162)$, Sự quen thuộc $(\beta=0.153)$, Các thông tin tiêu cực $(\beta=-0.110)$ và cuối cùng là Sự thu hút $(\beta=0.79)$. Phương trình hồi quy được trích theo hệ số beta chuẩn hóa như sau:

$$
\mathrm{YD}=0.482 \mathrm{PH}+0.199 \mathrm{TC}+0.162 \mathrm{CM}+0.153 \mathrm{QT}+(-0.110) \mathrm{TTTC}+0.079 \mathrm{TH} .
$$

\section{THẢO LUẬN KẾT QUẢ VÀ HÀM Ý QUẢN TRI}

\subsection{Thảo luận kết quả}

Kết quả nghiên cứu cho thấy có 6 yếu tố của đại sứ thương hiệu tác động đến ý định mua của khách hàng tại Thành phố Hồ Chí Minh. Trong đó, hệ số hồi quy của 5 biến độc lập Sự tin cậy, Sự thu hút, Chuyên môn, Sự quen thuộc, Sự phù hợp giữa đại sứ thương hiệu/sản phẩm đều lớn hơn 0 , hệ số mang dấu $(+)$, cho thấy, 5 biển độc lập này đều tác động cùng chiều tới đến ý định mua của khách hàng ở độ tin cậy $95 \%$ (sig $<0.05)$. Như vậy, các giả thuyết $\mathrm{H} 1, \mathrm{H} 2, \mathrm{H} 3, \mathrm{H} 4$ và $\mathrm{H} 5$ được chấp nhận. Còn biến độc các thông tin tiêu có sig $<0.05$ và hệ số hồi quy là -0.110 ,nghĩa là yếu tố các thông tin tiều có sự ảnh hưởng ngược chiều (-) đến đến ý định mua của khách hàng. Vì vậy, giả thuyết $\mathrm{H} 1, \mathrm{H} 2, \mathrm{H} 3, \mathrm{H} 4, \mathrm{H} 5, \mathrm{H} 6$ đều được chấp nhận. Sự phù hợp giữa đại sứ thương hiệu với thương hiệu và sản phẩm $(\beta=0.482)$ có ảnh hưởng cùng chiều với ý định mua của khách hàng. Kết quả này là phù hợp với các kết quả được công bố trước đây (Erdogan, 1999; Ha và Lam, 2016; Kalu, 2019; Farivar và cộng sự (2021). Sự tin cậy $(\beta=0.199)$ có ảnh hưởng cùng chiều lên ý định mua của khách hàng. Kết quả này phù hợp với kết quả nghiên cứu trước ở trong và ngoài nước (Ha và Lam, 2017; Giang và Dương, 2018; Osei-Frimpong và cộng sự; Weismueller và cộng sự, 2020). Chuyên môn $(\beta=0.162)$ có tác động cùng chiều đến ý định mua của khách hàng và kết quả này cũng tương thích với các công bố trước đây(Amos và cộng sự, 2008; Giang và Dương, 2018; Weismueller và cộng sự, 2020; Fernández và Castillo, 2021). Sự quen thuộc của đại sứ thương hiệu $(\beta=0.153)$ có ảnh hưởng cùng chiều đến ý định mua và kết quả này phù hợp với quan điểm của các nhà nghiên cứu trước đây (Ha và Lam, 2016; Osei-Frimpong và cộng sự, 2019; Utami và cộng sự , 2020; Fernández và Castillo, 2021). Sự thu hút $(\beta=0.79)$ của đại sứ thương hiệu cũng được chính minh là có tác động cùng chiều đến ý định mua của khách hàng và kết quả này là phù hợp với các kết quả được công bố trước đó (Giang và Dương, 2018; OseiFrimpong và cộng sự, 2019; Weismueller và cộng sự, 2020; Utami và cộng sự, 2020). Các thông tin tiêu cực $(\beta=-0.110)$ là yếu tố duy nhất trong mô hình có tác động nghịch chiều đến ý định mua. Kết quả này tương thích với các công bố trước đây về mối quan hệ nghịch chiều giữa thông tin tiêu cực của đại sứ thương hiệu và ý định mua của khách hàng (Thwaties và cộng sự, 2012; Prameswara và Sjabadhyni, 2018; Osei-Frimpong và cộng sự, 2019; Hafilah và cộng sự, 2019).

Dựa vào độ lớn của hệ số hồi quy chuẩn hóa beta, thứ tự mức độ tác động từ mạnh nhất tới yếu nhất của các biến độc lập đến biến phụ thuộc là: $\mathrm{PH}(0.482)>\mathrm{TC}(0.199)>\mathrm{CM}(0.162)>\mathrm{QT}(0.153)>\mathrm{TH}(0.079)$. Như vậy, Sự phù hợp giữa đại sứ thương hiệu/sản phẩm là yếu tố có tác động mạnh nhất đến ý định mua của khách hàng. Bên cạnh đó, ý định mua của khách hàng cũng bị ảnh hưởng ngược chiều bởi các thông tin tiêu cực từ phía đại diện thương hiệu. Từ kết quả nghiên cứu, các nhà quản trị marketing có thể sử dụng các hàm ý quản trị để nâng cao ý định mua hàng của khách hàng tại thành phố Hồ Chí Minh.

\subsection{Hàm ý quản trị}

Thứ nhất, sự phù hợp giữa thương hiệu và đại sứ thương hiệu (beta chuẩn hóa $=0.482$ ) là nhân tố tác động mạnh mẽ nhất đến ý định mua của người tiêu dùng. Do đó, các nhà quản trị marketing cần cân nhắc sự phù hợp giữa đại sứ thương hiệu với sản phẩm và thương hiệu. Sự đồng điệu giữa hình ảnh tích cực của đại sứ thương hiệu và thương hiệu công ty hay nhãn hàng sẽ giúp nâng cao sự thiện cảm nên người tiêu dùng; từ đó làm gia tăng ý định mua sản phẩm hay nhãn hàng mà đại sự đó đại diện quảng bá.

Thứ hai, kết quả nghiên cứu cho thấy sự tin cậy (beta chuẩn hóa $=0.199$ ) và chuyên môn (beta chuẩn hóa $=0.162$ ) của đại sứ thương hiệu là các nhân tố có tác động cùng chiều đến ý định mua của người tiêu dùng tại thành phố Hồ Chí Minh. Các công ty và nhãn hàng nên chọn đại sứ thương hiệu là chuyên gia hay là 
người có nhiều kinh nghiệm trong ngành hàng mà họ đại diện quảng bá. Khi đó, đại sứ thương hiệu mới đủ uy tín để thuyết phục khách hàng đặt niềm tin vào sản phẩm hay nhãn hàng mà họ đại diện quảng bá.

Thứ ba, sự quen thuộc của đại sự thương hiệu với người tiêu dùng (beta chuẩn hóa $=0.153$ ) cũng cần phải được cân nhắc cẩn thận khi chọn lựa đại sứ thương hiệu. Đối với các ngành hàng có mức độ phân khúc cao, người tiêu dùng có những đặc tính đặc thù thì sự quen thuộc của đại sự thương hiệu phải có độ gắn bó chặt chẽ với nhóm phân khúc mà họ đại diện để làm tăng sức ảnh hưởng của đại sứ thương hiệu lên ý định mua của người tiêu dùng. Sự thu hút của đại sứ thương hiệu cũng ảnh hưởng tích cực đến ý định người của khách hàng, đặt biệt là đối với những nhãn hàng phục vụ khách hàng trẻ tại thành phố Hồ Chí Minh. Các sản phẩm thuộc ngành hàng thời trang, mỹ phẩm, điện tử dân dụng thì các nhà quản trị marketing càng phải cân nhắc sự thu hút của đại sứ thương hiệu; đặc biệt là các đại sứ thương hiệu có lượng người hâm mộ đông đảo để lưạ chọn đại sứ thương hiệu cho phù hợp.

Thứ tư, hàm ý quản trị sau cùng là phải hạn chế các thông tin tiêu cực (beta chuẩn hóa =-0.11) của đại sứ thương hiệu đến hình ảnh của sản phẩm hay công ty mà họ đại diện quảng bá. Các đại sự thương hiệu và công ty cần phải ký kết càng các điều khoản trong hợp đồng pháp lý để quy định các hành động và phát ngôn như thế nào là không phù hợp. Hậu quả là làm xuất hiện các thông tin tiêu cực gây ảnh hưởng xấu đển giá trị thương hiệu của sản phẩm hay công ty. Ngoài ra, các nhà quản trị marketing cũng cần phải chuẩn bị các kế hoạch xử lý khủng hoảng nếu xuất hiện các thông tin tiêu cực của đại sứ thương hiệu nhằm giảm bớt ảnh hưởng xấu của những thông tin này lển ý định mua của khách hàng.Thực hiện các nhóm hàm ý quản trị này một cách nhất quán và chặt chẽ sẽ giúp đại sứ thương hiệu và các nhãn hàng đạt được những mục tiêu của hai bên trong việc duy trì và phát huy hình ảnh tích cực đối với khách hàng tại thành phố Hồ Chí Minh.

\subsection{Hạn chế và hướng nghiên cứu tiếp theo}

Bên cạnh những ý nghĩa và đóng góp của nghiên cứu, nghiên cứu này vẫn còn tồn tại một số hạn chế nhất định.Thứ nhất, nghiên cứu thu thập dữ liệu từ mẫu chủ yếu là đối tượng là người trẻ, bằng phương pháp lấy mẫu thuận tiện, số lượng mẫu chưa đủ lớn nên chỉ có thể đại diện cho một phần nhỏ người tiêu dùng tại TP.Hồ Chí Minh. Do đó, kết quả nghiên cứu chưa thể đánh giá tổng quát hết được đánh giá của khách hàng về sự tác động của các yếu tố của đại sứ thương hiệu đến ý định mua hàng.Thứ hai, thời gian nghiên cứu có hạn nên có sự hạn chế khi tìm kiếm tài liệu để xây dựng mô hình và thang đo phù hợp nhất cũng như là hạn chế trong việc thu thập số lượng mẫu khảo sát nên cũng ảnh hưởng đến kết quả phân tích. Thứ ba, đề tài nghiên cứu ở Việt Nam còn mới nên tài liệu nghiên cứu chủ yếu đến từ nước ngoài nên có nhiều khó khăn khi thu thập tài liệu để phân tích và tham khảo các kết quả nghiên cứu trước đây. Các hướng nghiên cứu trong tiếp theo có thể cố gắng phân tích dựa trên một số nhân vật đại sứ thương hiệu cụ thể để phân biệt và so sánh những ảnh hưởng của đại sứ thương hiệu tiền nhiệm và hiện tại bằng cách đưa những đại sứ thương hiệu tiền nhiệm và hiện tại của thương hiệu vào nghiên cứu. Ngoài ra, các nhà nghiên cứu có thể tìm hiểu thêm một số nhân tố, biến kiểm soát khác hơn và thêm biến quan sát mới vào mô hình và thang đo. Bên cạnh đó, sử dụng phương pháp lấy mẫu ngẫu nhiên (đơn giản hoặc có hệ thống) từ những nguồn khác nhau nhằm gia tăng khả năng khái quát hóa của nghiên cứu.

\section{TÀI LIỆU THAM KHẢO}

Adage. (2021). Retrieved 14 July 2021, from https://adage.com/article/cmo-strategy/regularly-updated-blog-

tracking-brands-responses-racial-injustice/2260291.

Ajzen, I. (1991). The Theory of Planned Behavior. Organizational Behavior and Human Decision Processes, 50(2), 179-211.

Alagoz, S. M., \& Hekimoglu, H. (2012). A study on TAM: Analysis of customer attitudes in online food. Procedia Social and Behavioral Sciences, 62, $1138-1143$.

Alba, Joseph W. and J. Wesley Hutchinson (1987), "Dimensions of Consumer Expertise," Journal of Consumer Research, 13 (March), 411-454.

Antunes, A. C. (2021). The Role of Social Media Influencers on the Consumer Decision-Making Process. In Analyzing Global Social Media Consumption (pp. 138-154). IGI Global. 
Brown, S. A., \& Venkatesh, V. (2005). Model of Adoption of Technology in the Household: A Baseline Model Test and Ext, Incorporating Household Life Cycle. MIS Quarterly, 29(4), 399-426.

Chen, C-F, \& Chao, W-S. (2010). Habitual or reasoned? Using the theory of planned behavior, technology acceptance model, and habit to examine switching intentions toward public transit. Transportation Research Part F: Traffic Psychology and Behaviour, 14(2), 128-137.

Chen, X., Kim, K., Yao, T., \& Yu, T. (2010). On the predictability of Chinese stock returns. Pacific-Basin Finance Journal, 18(4), 403-425.

Chuttur, M. Y. (2009). Overview of the Technology Acceptance Model: Origins, Developments and Future Directions. Sprouts: Working Papers on Information Systems, 9(37).

Cục Thương mại điện tử và Kinh tế số. (2020). Thương mại điện tử Việt Nam. Bộ Công thương.

Darke, P. R., \& Ritchie, R. J. (2007). The defensive consumer: Advertising deception, defensive processing, and distrust. Journal of Marketing research, 44(1), 114-127.

Davis, F. D. (1989, September). Perceived usefulness, perceived ease of use, and user acceptance of information technology. MIS Quarterly, 13(3), 319-340.

Davis. (1989). Technology Acceptance Model.

Degeratu, A., Rangaswamy, A., \& Wu, J. (2000). Consumer choice behavior in online and traditional supermarkets: The effects of brand name, price, and other search attributes. International Journal of Research in Marketing, 17(1), 55-78.

Digital Marketing Institute. (2021). 20 Surprising Influencer Marketing Statistics | DMI. Retrieved 16 June 2021, from https://digitalmarketinginstitute.com/blog/20-influencer-marketing-statistics-that-will-surprise-you

Dodds, W. B., Monroe, K. B., \& Grewal, D. (1991). Effects of Price, Brand and Store Information for Buyers. Journal of Marketing Research, 28(3), 307-319.

Erdogan, B. (1999). 'Celebrity Endorsement: A Literature Review'. Journal of Marketing Management, 15(4), pp.291-314. doi: 10.1362/026725799784870379.

Esangbedo, R. (2011). The impact of celebrity endorsements on the buying behavior of Rowan University students age 18-24.

Farivar, S., Wang, F., \& Yuan, Y. (2021). Opinion leadership vs. para-social relationship: Key factors in influencer marketing. Journal of Retailing and Consumer Services, 59, 102371.

Fishbein, M., \& Ajzen, I. (1976). Misconceptions about the Fishbein model: Reflections on a study by SongerNocks. Journal of Experimental Social Psychology, 12(6), 579-584.

Giang, N. T., \& Dương, P. T. (2018). Nhận thức của người tiêu dùng về đại diện thương hiệu là người nổi tiếng. Tạp chí Khoa học \& Đào tạo Ngân hàng , 54-63.

Giao, H. N. K., \& Trà, B. T. (2020). Quyết định mua vé máy bay trực tuyến của người tiêu dùng TPHCM.

Ha, N. M., \& Lam, N. H. (2017). The effects of celebrity endorsement on customer's attitude toward brand and purchase intention. International Journal of Economics and Finance, 9(1), 64-77.

Habing. (2003). (Prentical-Hall International, Inc, Ed.) Exploratory Factor Analysis.

Hair et al. (2006, January). Mutivariate Data Analysis. (3, Ed.) Technometrics, 31.

Hair, Joseph F.; Hult, G. Thomas M.; Ringle, Christian; and Sarstedt, Marko. (2014). A Primer on Partial Least Squares Structural Equation Modeling (PLSSEM). Thousand Oaks,CA: Sege.

Hoàng Trọng \& Chu Nguyễn Mộng Ngọc . (2008). Phân tích dũ liệu nghiên cưu với SPSS, Tập 1, Tập 2. NXB Hồng Đức.

Hoàng Trọng, \& Chu Nguyễn Mộng Ngọc. (2008). Phân tích dũ liệu nghiên cúu với SPSS Tập 2. NXB Hồng Đức.

Hsieh, M.-H., Pan, S.-L., \& Setiono, R. (2004). Product-, corporate-, and country-image dimensions and purchase behavior: A multicountry analysis. Journal of the Academy of Marketing Science, 32(3), 251-270. 
Huang, W.-y., Schrank, H., \& Dubinsky, A. (2004). Effect of brand name on consumers' risk perceptions of online shopping. Journal of Consumer Behaviour, 4(1), 40-50.

Huynh, V. (2020). The Role of Social Media Influencers in the Consumer Decision-making Process.

Jara, A. J., Parra, M. C., \& Skarmeta, A. F. (2012, July). Marketing 4.0: A new value added to the Marketing through the Internet of Things. In 2012 Sixth International Conference on Innovative Mobile and Internet Services in Ubiquitous Computing (pp. 852-857). IEEE.

Järvinen, R., \& Lehtinen, U. (2004). Services, e-services and e-service innovations - Combination of theoretical and practical knowledge. Frontiers of e-Business Research, 84.

Jeong, C. H. (2007). Fundamental of Development Administration. Selangor: Scholar Press.

Kalu, F. (2019). The Impact Of Social Media Influencer Marketing On Purchase Intention From An Irish Male Millennial's Perception: A Case Study Of Irish Fashion Industry(Doctoral dissertation, Dublin, National College of Ireland).

Kent, Robert J. and Chris T. Allen (1994), “Competitive Interfer- ence Effects in Consumer Memory for Advertising: The Role of Brand Familiarity," Journal of Marketing, 58 (July), 97-105.

Kết quả xuất nhập khẩu trong thời gian qua và công tác điều hành xuất nhập khẩu của Bộ Công Thuong. (2020, 10 24). From Bộ Công Thương Việt Nam: https:/www.moit.gov.vn/web/guest/tin-chi-tiet/-/chi-tiet/ket-quaxuat-nhap-khau-trong-thoi-gian-qua-va-cong-tac-\%C4\%91ieu-hanh-xuat-nhap-khau-cua-bo-cong-thuong20696-22.html

Kirmani, A. (2009). The self and the brand. Journal of Consumer Psychology, 19(3), 271-275.

Knott, C. L., \& James, M. S. (2004). An alternate approach to developing a total celebrity endorser rating model using the analytic hierarchy process. International transactions in operational research, 11(1), 87-95.

Kotler, P. (2009). Marketing management: A south Asian perspective. Pearson Education India.

Kotler, P., Kartajaya, H., \& Setiawan, I. (2017). Marketing 4.0. Moving from Traditional to Digital, Published by John Wiley\&Sons. Inc., Hoboken, New Jersey, 32.

Leeflang, P. S., Verhoef, P. C., Dahlström, P., \& Freundt, T. (2014). Challenges and solutions for marketing in a digital era. European management journal, 32(1), 1-12.

Ngân, H. T. K. (2018). Nghiên cứu các yếu tố ảnh hưởng đến ý định mua sắm trực tuyến của người tiêu dùng đối với hàng nông sản.

Nguyễn Đình Thọ. (2011). Phương pháp nghiên cứu khoa học trong kinh doanh. NXB Lao động Xã hội.

Nguyễn Thị Ngọc Oanh. (2016). Các yếu tố ảnh hưởng đến ý định áp dụng EMA tại các doanh nghiệp sản xuất khu vục phía Nam Việt Nam.

Nguyễn, B. (2021). Hoạt động kinh doanh bảo hiểm phi nhân thọ tại Việt Nam. Retrieved 16 June 2021, from https://tapchicongthuong.vn/bai-viet/van-dung-ly-thuyet-hanh-vi-nguoi-tieu-dung-trong-hoat-dong-kinh-doanh-baohiem-phi-nhan-tho-tai-viet-nam-trong-giai-doan-hien-nay-75614.htm

Nguyễn, T., \& Lê, Đ. (2021). CÁC NHÂN TỐ ẢNH HƯỎNG ĐẾN Ý ĐỊNH MUA SẮM MỸ PHẨM ORGANIC CỦA NGƯỜI TIÊU DÙNG TRÊN ĐỊA BÀN THÀNH PHỐ HỒ CHÍ MINH. Retrieved 16 June 2021, from https://tapchi.ftu.edu.vn/các-số-tạp-ch\%C3\%AD-ktđn/184-tạp-ch\%C3\%AD-ktđn-số-110-đến-số-119/tạpch\%C3\%AD-ktđn-số-116/1586-các-nhân-tố-ảnh-hưởng-đến-ý-định-mua-sắm-mỹ-phẩm-organic-của-người-tiêudùng-trên-địa-bàn-thành-phố-hồ-ch\%C3\%AD-minh.html.

Oanh, N. T. N. (2016). Các nhân tố ảnh hưởng đến ý định áp dụng kế toán quản trị môi trường (EMA) tại các doanh nghiệp sản xuất khu vực phía nam Việt Nam.

Osei-Frimpong, K., Donkor, G., \& Owusu-Frimpong, N. (2019). The impact of celebrity endorsement on consumer purchase intention: An emerging market perspective. Journal of marketing theory and practice, 27(1), 103121. 
Phương, D. T. (2012). Nghiên cứu các nhân tố ảnh hưởng đến ý định mua sắm trực tuyến của khách hàng trên địa bàn thành phố Huế. Chuyên san khoa học Xã hội và Nhân văn, 72(3).

Prameswara, D. H., \& Sjabadhyni, B. (2018, July). The Effect of Negative Celebrity Publicity and Celebrity Identification Towards Purchase Intention. In Universitas Indonesia International Psychology Symposium for Undergraduate Research (UIPSUR 2017) (pp. 59-66). Atlantis Press.

Roger. (1983). The Diffusion of Innovation Theory.

Rowley, J. (2006). An analysis of the e-service literature: towards a research agenda. Internet Research, 16(3), 339359.

Rust, R. T., \& Kannan, P. K. (2002). e-Service: New Direction in Theory and Practice. Armonk, New York: M.E. Sharpe.

Sánchez-Fernández, R., \& Jiménez-Castillo, D. (2021). How social media influencers affect behavioural intentions towards recommended brands: the role of emotional attachment and information value. Journal of Marketing Management, 1-25.

Scott, D. M. (2015). The new rules of marketing and PR: How to use social media, online video, mobile applications, blogs, news releases, and viral marketing to reach buyers directly. John Wiley \& Sons.

Thanh, N. L. P. (2013). Các yếu tố ảnh huơơng đến ý định mua sắm trực tuyến của khách hàng tại Việt Nam (Doctoral dissertation, Luận văn thạc sĩ, Đại học Kinh tế Thành phố Hồ Chí Minh).

Thwaites, D., Lowe, B., Monkhouse, L. L., \& Barnes, B. R. (2012). The impact of negative publicity on celebrity ad endorsements. Psychology \& Marketing, 29(9), 663-673.

Todor, R. D. (2014). The importance of branding and rebranding for strategic marketing. Bulletin of the Transilvania University of Brasov. Economic Sciences. Series V, 7(2), 59.

Utami, S. P., Setyowati, N., \& Mandasari, P. (2020). Celebrity Brand Ambassador and e-WOM as Determinants of Purchase Intention: A Survey of Indonesian Celebrity Cake. In E3S Web of Conferences (Vol. 142, p. 05001). EDP Sciences.

Venkatesh, V. (1999, 2). A Theoretical Extension of the Technology Acceptance Model: Four Longitudinal Field Studies. 46(2), 186-204.

Venkatesh, V., Morris, M. G., Davis, G. B., \& Davis, F. D. (2003). User Acceptance of Information Technology: Toward a Unified View. MIS Quarterly, 27(3), 425-478.

Venkatesh, V., Thong, J. Y., \& Xu, X. (2012). Consumer acceptance and use of information technology: Extending the Unified theory of acceptance and use of technology. MIS Quarterly, 36(1), 157-178.

Weismueller, J., Harrigan, P., Wang, S., \& Soutar, G. N. (2020). Influencer endorsements: How advertising disclosure and source credibility affect consumer purchase intention on social media. Australasian marketing journal, 28(4), 160-170.

Wills, P. (2021). Mere Exposure Effect. In Decision Making in Emergency Medicine (pp. 209-213). Springer, Singapore.

Ngày nhận bài: 11/05/2021

Ngày chấp nhận đăng: 20/07/2021 